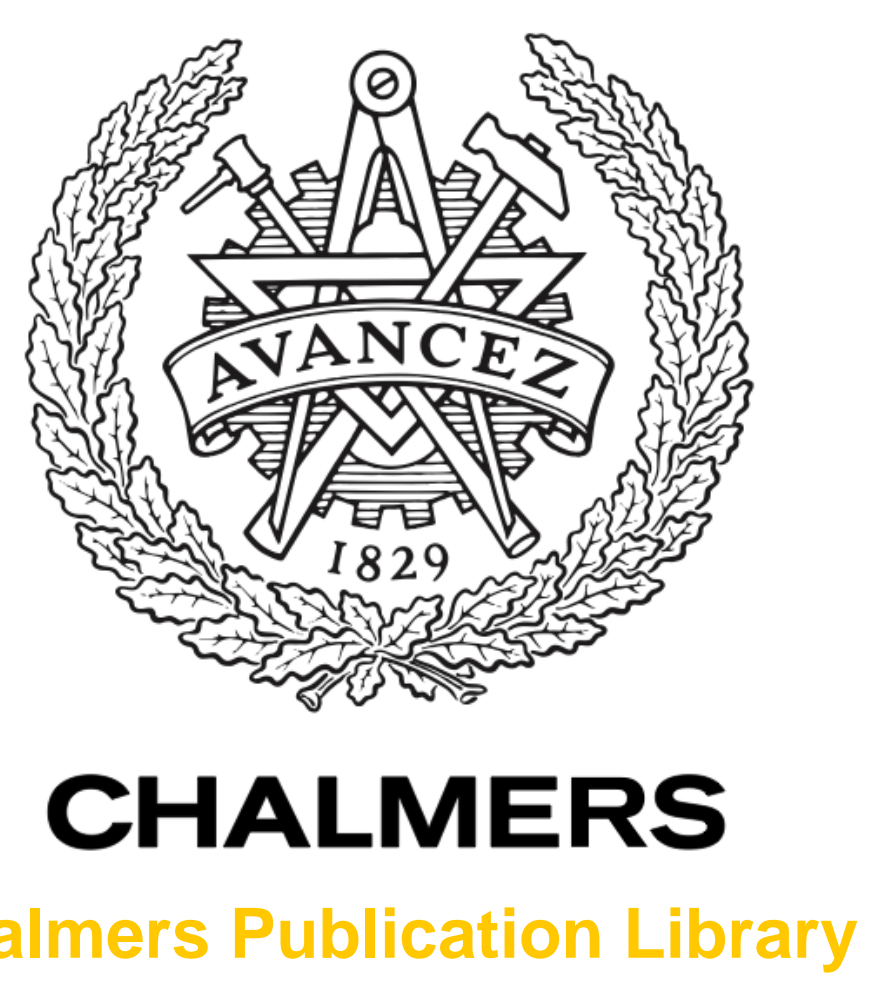

Chalmers Publication Library

\title{
Assessment of Ground-BasedMicrowave Radiometry for Calibration of Atmospheric Variability in Spacecraft Tracking
}

This document has been downloaded from Chalmers Publication Library (CPL). It is the author's version of a work that was accepted for publication in:

IEEE Transactions on Antennas and Propagation (ISSN: 0018-926X)

Citation for the published paper:

Graziani, A. ; Jarlemark, P. ; Elgered, G. (2014) "Assessment of Ground-BasedMicrowave Radiometry for Calibration of Atmospheric Variability in Spacecraft Tracking". IEEE

Transactions on Antennas and Propagation, vol. 62(5), pp. 2634-2641.

http://dx.doi.org/10.1109/TAP.2014.2307582

Downloaded from: http://publications.lib.chalmers.se/publication/197519

Notice: Changes introduced as a result of publishing processes such as copy-editing and formatting may not be reflected in this document. For a definitive version of this work, please refer to the published source. Please note that access to the published version might require a subscription.

Chalmers Publication Library (CPL) offers the possibility of retrieving research publications produced at Chalmers University of Technology. It covers all types of publications: articles, dissertations, licentiate theses, masters theses, conference papers, reports etc. Since 2006 it is the official tool for Chalmers official publication statistics. To ensure that Chalmers research results are disseminated as widely as possible, an Open Access Policy has been adopted.

The CPL service is administrated and maintained by Chalmers Library. 


\title{
Assessment of Ground-Based Microwave Radiometry for Calibration of Atmospheric Variability in Spacecraft Tracking
}

\author{
A. Graziani, P. Jarlemark, G. Elgered, A. Martellucci, M. Mercolino and P. Tortora, Member, IEEE
}

\begin{abstract}
In a suggested radio propagation experiment using a Deep Space Antenna, accurate calibration of the propagation delay through the Earth's atmosphere is essential. One or two microwave radiometers can be used for this purpose. Differences in precise locations of the radiometer(s) and antenna to be calibrated leave a residual wet path delay value. We computed the Allan Standard Deviation (ASD) of this residual, as well as the one resulting from different pointing positions in the plane of the sky, by simulations. Pointing offsets, e.g. to avoid solar radiation into the radiometer beam, lead in general to an increased ASD. However, for many observation geometries a deliberate pointing offset can compensate for the location differences. In the case studied we found a reduction of the ASD with up to $45 \%$ compared to the ASD obtained for a zero pointing offset. The size of the calculated ASD depends strongly on the model parameters used, e.g. the turbulence strength parameter $\mathrm{C}_{\mathrm{n}}{ }^{2}$, which has a significant natural variation over a year.
\end{abstract}

Index Terms-Atmospheric modeling, Microwave Radiometry, Space exploration

\section{INTRODUCTION}

A DEDICATED Media Calibration System (MCS) is a crucial tool in order to estimate the atmospheric path delay along the line-of-sight (LOS) from the tracked probe to the ground station in demanding radio science experiments [1]. An MCS is made up from a combination of different meteorological instruments used to retrieve the atmospheric path delay.

Manuscript received September 1, 2013. This work was funded by the ESA contract 23127/10 named AWARDS: Advanced Microwave Radiometer in Deep Space Station. The work of Alberto Graziani and Paolo Tortora was also funded in part by the Italian Space Agency (ASI) through contract $\mathrm{I} / 080 / 09 / 0$.

A. Graziani is with the Department of Industrial Engineering of the University of Bologna, Forlì, Italy (alberto.graziani@ unibo.it).

P. Jarlemark is with the SP Technical Research Institute of Sweden, Borås, Sweden (per.jarlemark@sp.se).

G. Elgered is with the Department of Earth and Space Sciences of the Chalmers University of Technology, Gothenburg, Sweden (gunnar.elgered@chalmers.se)

A. Martellucci is with the European Space Agency, ESA/ESTEC, Noordwijk, The Netherlands, (antonio.martellucci@esa.int)

M. Mercolino is with the European Space Agency ESA/ESOC, Darmstadt, Germany (mattia.mercolino@esa.int)

P. Tortora is with the Department of Industrial Engineering of the University of Bologna, Forlì, Italy (paolo.tortora@unibo.it).
This work can be placed in the framework of the forthcoming Bepi-Colombo mission, of the European Space Agency (ESA), to Mercury [2], and in particular in the evaluation of the MCS to calibrate the deep space observables for the Mercury Orbiter Radioscience Experiment (MORE) [3]. MORE encompass a set of challenging experiments to investigate a wide range of physical parameters, spanning in the fields of geodesy, geophysics and fundamental physics: it includes the determination of the gravity field of Mercury, the properties and the topography of Mercury's surface (in combination with the laser altimeter instrument BELA), but also the internal structure of the planet and its rotational state. Moreover, during the cruise phase of the mission, MORE will probe fundamental physics theories, with the most precise experimental estimation of some of the Post-Newtonian parameters. In order to achieve the expected experiment results, all error sources need to be suppressed or mitigated at a desired level. One of the main error sources is the propagation path delay caused by the neutral atmosphere which, due to its non-dispersive nature, cannot be canceled out by the use multifrequency radio links, necessary to compensate for solar and interplanetary plasma. For this reason, it is necessary to use additional dedicated instruments on the ground.

Many techniques are available to estimate different parameters in order to characterize atmospheric variability, e.g., in situ measurements with high resolution radiosondes [4] ground-based radar systems [5], radio interferometry [6], ground-based GPS receivers [7] [8] and MWRs. The short term variations in the propagation delay are dominated by water vapor in the troposphere. These variations, which are sometimes referred to as atmospheric turbulence, can be studied by several techniques, e.g. RADAR [9], LIDAR [10] and SCIDAR [11]. In our application, the integrated effect along an Earth-space path is the fundamental parameter. The most attractive instrument is a microwave radiometer (MWR), capable of estimating the "wet" contribution of the atmospheric path delay along the LOS to the space probe [12]. When the wet delay has been corrected using MWR observations the variations in the dry refractivity (mainly temperature) and the hydrostatic delay [13] may become an equally important error source. One evident aspect of the MCS is the amount of instrumental noise that will encompass the calibration data. This is not addressed in this paper but an 
extensive review of the MWR stability requirements for MORE is given in [14]. Another important aspect of the MCS, and in particular the MWR, is its position with respect to the space probe tracking antenna, referred to as the Deep Space Antenna (DSA) following the naming convention of ESA. We assume that one or two MWRs are installed close to, and not mounted on, the DSA due to the complexity and the maintenance aspects of the entire system. For this reason, the atmospheric volumes observed by the two systems (DSA and MWR) will always be different. In order to evaluate the quality of the calibration caused by different geometries, the effect of the atmospheric fluctuations has to be assessed.

For the MORE error budget the contributions are described in terms of the Allan Standard Deviation (ASD) [15], a parameter that can be used to estimate the standard deviation of processes with temporal drifts. A common definition is:

$$
\operatorname{ASD}(\tau)=\left(\left\langle\frac{(x(t+2 \tau)-2 x(t+\tau)+x(t))^{2}}{2 \tau^{2}}\right\rangle\right)^{1 / 2}
$$

Where $x(t)$ is the process (e.g. the error in the wet delay calibration), $t$ is the time, $\tau$ is a time separation, in our application related to the integration time defined as the interval over which Doppler tracking observables are averaged to reduce background noise and increase the signal-to-noise ratio. The brackets $<>$ denote expectation value.

The maximum contributions from the residual troposphere (after calibration) allowed in the MORE error budget are reported in Table I, where different integration times are defined in order to have reference values for both deep-space ranging and Doppler observables. On the other hand, the same values are used as design requirements for the definition of the studied MCS [16].

TABLE 1.

RADIOSCIENCE EXPERIMENT REQUIREMENTS

\begin{tabular}{|c|c|c|c|}
\hline Integration Time $[\mathbf{s}]$ & 20 & 1000 & 10000 \\
\hline ASD $[\mathbf{s} / \mathbf{s}]$ & $3 \times 10^{-14}$ & $3 \times 10^{-15}$ & $3 \times 10^{-15}$ \\
\hline
\end{tabular}

There are three main different effects that need to be simulated: (A) the differences between the true beam shapes, of the DSA and the MWR antennas, vs. a pencil beam; (B) the site position offset(s) between the DSA and the MWR(s) on the ground, and (C) the pointing offsets in the plane of the sky. Tortora et al. [16] studied these effects and found the following. The effect due to antenna beams is small and actually it reduces the residual ASD by $10-20 \%$ due to averaging of the variations within the volume of air sensed by the antennas. A realistic and reasonable position offset is of the order of $25 \mathrm{~m}$. A pointing offset of the MWR away from the DSA shall be kept to a minimum.

In this paper we will simulate the combination of these three effects using a number of different geometries (see Fig. 1 for an example). In Section II we present the model used for the atmospheric variability. Section III presents simulation results for a standard configuration, observations in a direction close to the sun, and mitigation obtained when the beams of the DSA and the MWR(s) are crossing. The conclusions are presented in Section IV.

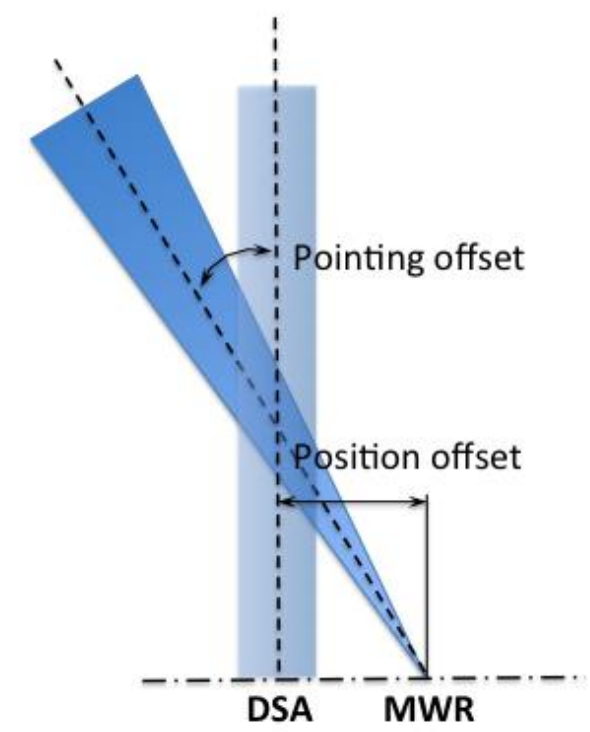

Fig. 1 An illustration of a possible configuration of the MWR beam with respect to the DSA beam.

\section{Modeling AtMospheric VARIABILITY}

A methodology for statistical representation of wet delay differences based on atmospheric parameters is given in [17]. Included in this modeling is the "frozen flow hypothesis": the statistics of spatial variations in the atmosphere can be transformed to temporal variation statistics by assuming that spatial variations are propagating over a site with a characteristic wind velocity. Fig. 2 depicts the expected ASD of the uncalibrated wet delay for different geometries according to this model for different observational directions. At lower elevation angles the longer observation paths through the atmosphere in general lead to greater ASD values. When observing in azimuth directions along the wind vector the distance in the propagating medium between the paths at two time instants is smaller than the path distance for observations perpendicular to the wind vector. This leads to smaller ASD values at zero azimuth offsets as seen for shorter time separations in Fig. 2. However, at longer time separations this azimuth effect on the distance is insignificant. 


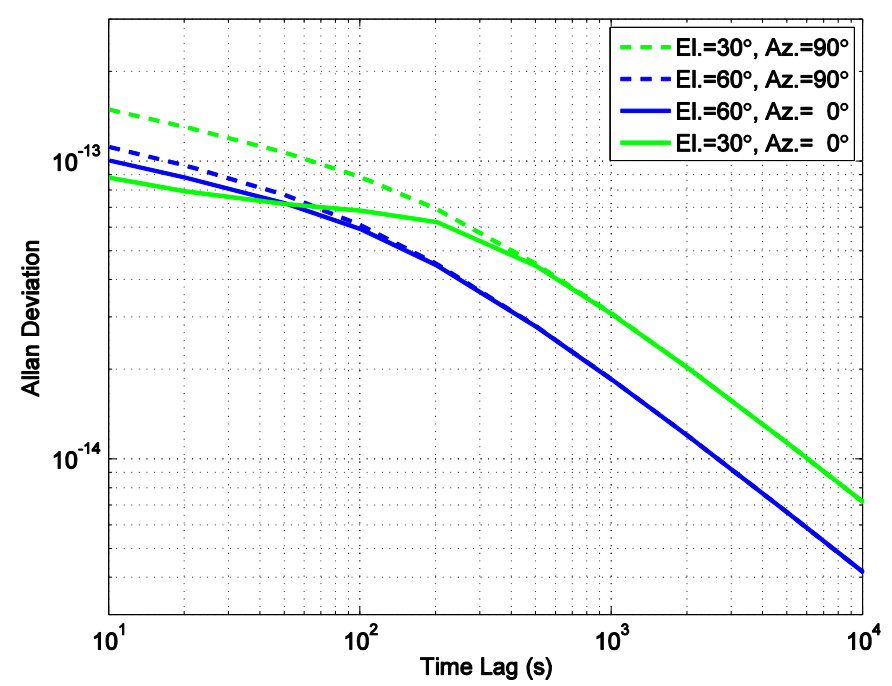

Fig. 2 The ASD of the uncalibrated wet delay at different elevation angles (El) and azimuth angles $(\mathrm{Az})$. Azimuth angles are given relative to the wind direction. The legend reflects the curve order top-down at $10 \mathrm{~s}$ integration time.

In this study we have used the modeling and parameter values presented in [17] for calculating the ASD of the residual wet delay when the DSA is calibrated using data from one or two ideal MWR(s). Here we summarize the equations for the geometry of using one MWR. The theory for the case when two MWRs are used is along the same lines, see [18].

With $l_{D}$ and $l_{M}$ denoting the wet delay at the DSA and a MWR, respectively, we can form the ASD for the residual delay, $\Delta l=l_{M}-l_{D}$, replacing $x(t)$ in (1) and obtain:

$$
A S D_{\Delta l}(\tau)=\left(\left\langle\frac{(\Delta l(t+2 \tau)-2 \Delta l(t+\tau)+\Delta l(t))^{2}}{2 \tau^{2}}\right\rangle\right)^{1 / 2}
$$

The numerator in the fraction of (2) can be expanded into terms with pairs of wet delays.

$$
\begin{aligned}
& \left\langle\left[\left(l_{M}(t+2 \tau)-l_{D}(t+2 \tau)\right)-2\left(l_{M}(t+\tau)-l_{D}(t+\tau)\right)+\left(l_{M}(t)-l_{D}(t)\right)\right]^{2}\right\rangle= \\
& 6\left\langle\left[l_{M}(t)-l_{D}(t)\right]^{2}\right\rangle+4\left\langle\left[l_{M}(t+\tau)-l_{M}(t)\right]^{2}\right\rangle-4\left\langle\left[l_{M}(t+\tau)-l_{D}(t)\right]^{2}\right\rangle \\
& -4\left\langle\left[l_{D}(t+\tau)-l_{M}(t)\right]^{2}\right\rangle+4\left\langle\left[l_{D}(t+\tau)-l_{D}(t)\right]^{2}\right\rangle-\left\langle\left[l_{M}(t+2 \tau)-l_{M}(t)\right]^{2}\right\rangle \\
& +\left\langle\left[l_{M}(t+2 \tau)-l_{D}(t)\right]^{2}\right\rangle+\left\langle\left[l_{D}(t+2 \tau)-l_{M}(t)\right]^{2}\right\rangle-\left\langle\left[l_{D}(t+2 \tau)-l_{D}(t)\right]^{2}\right\rangle
\end{aligned}
$$

In (3) we have assumed stationary of the statistics, i.e., independence of $t$.

Each term on the right hand side of (3) can now be calculated according to [17] using the model for differences in the refractive index, $n$, at two locations $r_{1}$ and $r_{2}$ :

$$
\left\langle\left[n\left(\vec{r}_{1}, t\right)-n\left(\vec{r}_{2}, t+\Delta t\right)\right]^{2}\right\rangle=C_{n}^{2} d^{2 / 3}
$$

where the "frozen flow" distance $d$ is defined as

$$
d=\left|\vec{r}_{2}+\Delta t \cdot \vec{v}-\vec{r}_{1}\right|
$$

Where $v$ is the wind vector and we refer to the constant $C_{n}{ }^{2}$ as the turbulence strength parameter.

For the investigated pointing offset configuration of the DSA and MWR antennas the simulation performed consist of parametrizing the position vectors $r_{1}$ and $r_{2}$ along the lines of sight for the ray pair in each term in (3). In all calculations the standard values of the parameters presented in [17] have been used. Among these are $C_{n}^{2}$ equal to $5.7610^{-14} \mathrm{~m}^{-2 / 3}$, the height of the layer with constant turbulence is $1 \mathrm{~km}$, and the wind velocity is $8 \mathrm{~m} / \mathrm{s}$.

For practical reasons the results have been calculated using pencil beam models for the DSA and MWR(s). However, the extensions of the actual antenna beams studied lead to spatial averaging of the measured wet delay. Hence the ASD of the residual wet delay measured is 0.8-0.9 times the ASD calculated for the pencil shaped beams [16]. We have therefore multiplied the pencil beam results with $\sim 0.85$ in order to compensate for the expected spatial averaging.

\section{Simulation OF DifFERENT CONFIGURATIONS}

The ASD of the residual wet delay was computed for a set of geometrical configurations of position and pointing offsets that the DSA and MWR(s) may encounter.

For the computed ASD of the uncalibrated wet delay the modeled wind direction has a large influence, as shown in Fig. 2. However, for the residual ASD calculations presented in this section the wind directions have a much less significant role, of the order of $10 \%$. The direction of the baseline between DSA and MWR has a more significant influence on the residual wet delay ASD. In all simulations presented below the azimuth angle of the wind vector has been set to $45^{\circ}$.

\section{A. Standard configuration}

Configurations with both one and two MWR(s) for calibration of the DSA wet delay are studied using simulations. The MWR(s) are situated $25 \mathrm{~m}$ from the DSA; in the one-MWR case south of the DSA, while in the two-MWR case one to the south-east and one to the south-west with a separation of $20 \mathrm{~m}$ between the MWRs. In the standard configuration the MWR pointing direction is identical with the DSA pointing. Using the variability model presented above, the residual ASD after calibration with ideal MWR data are calculated for a set of pointing directions. The results for pointing to the east and south at different elevation angles are presented in Fig. 3 and Fig. 4, respectively. Included in the graphs are also the earlier stated requirements on the calibration. 


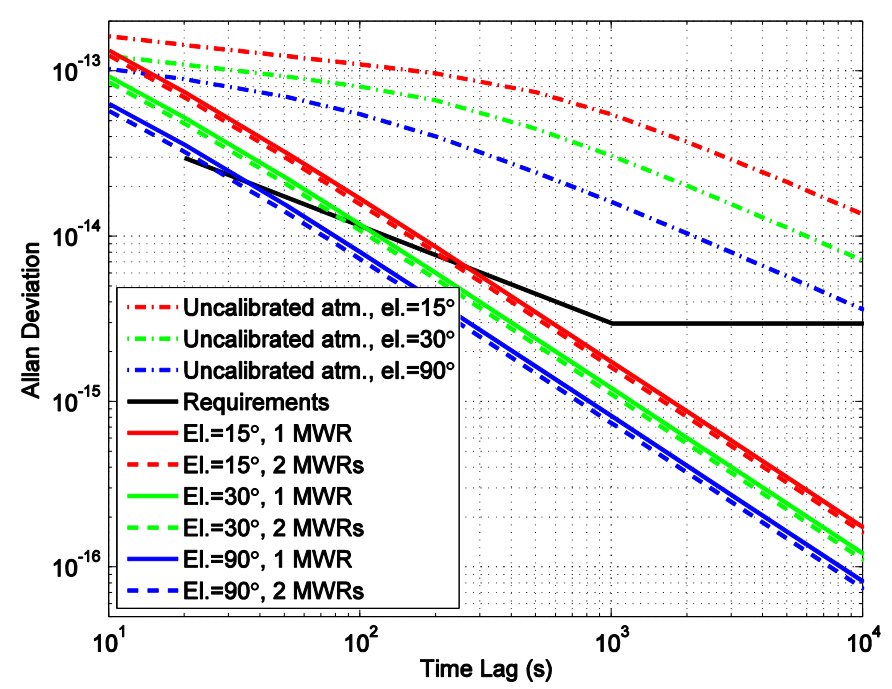

Fig. 3 The residual ASD when the wet delay of the DSA is calibrated using data from 1 or 2 MWR(s) located $25 \mathrm{~m}$ to the south of the DSA and pointing the antennas in the east direction. Curves for the uncalibrated ASD of the wet delay in the observation directions, as well as the design requirements have been added for comparison. The legend reflects the curve order top-down at 1000 s integration time.

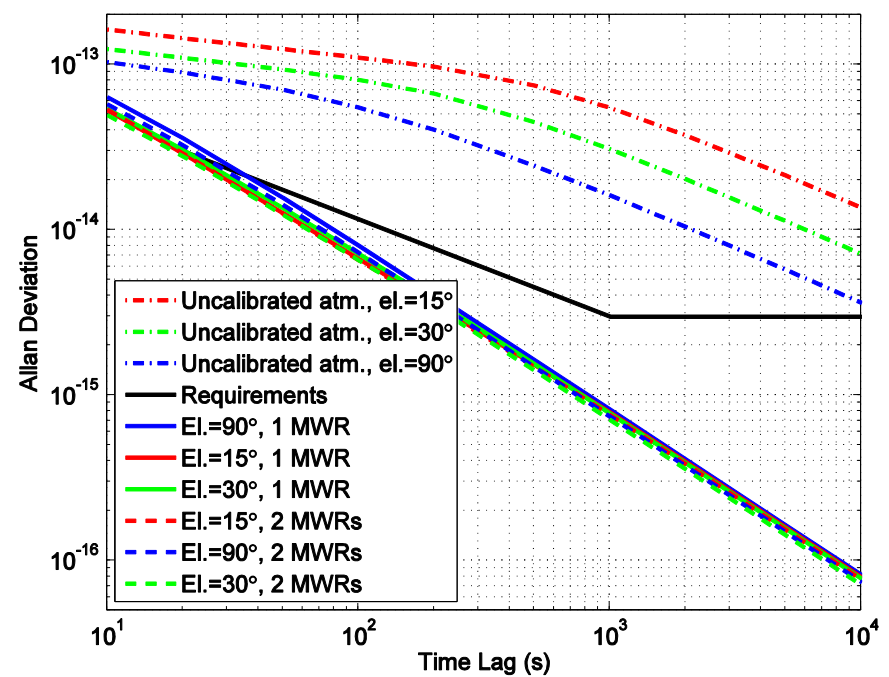

Fig. 4 The residual ASD when the wet delay of the DSA is calibrated using data from 1 or 2 MWR(s) located $25 \mathrm{~m}$ to the south of the DSA and pointing the antennas in the south direction. Curves for the uncalibrated ASD of the wet delay in the observation directions, as well as the design requirements have been added for comparison. The values for the calibrated wet delay are so similar that not all curves are distinguishable. The legend reflects the curve order top-down at $1000 \mathrm{~s}$ integration time.

It is seen that the requirements for longer integration times are relatively easily met according to the model used. However, at shorter time scales, say 20-200 s, the presented design could be expected to fail the requirement under many observational conditions. There is a reduction in the residual ASD when using 2 MWRs instead of one, but it is at most approximately $10 \%$ for the observational geometries presented. Ideally, the gain from using dual MWRs in this standard configuration could be larger if the distance between them was greater, and thereby their observed air volumes differed more. This would, however, lead to moving the MWRs away from their positions south of the DSA and, in order to remain with the same distance from the DSA, lead to higher risk for the DSA dish to interfere with the MWR observations.

One interesting feature of Fig. 4 is the small ASD difference between observations at different elevation angles when pointing to the south. The longer observation paths through the atmosphere at lower elevation angles would have the potential to yield greater ASD values. However, when observing with the DSA pointing in the azimuth direction of the MWR(s) the actual distance between the DSA and MWR paths decrease at lower elevation angles, thereby compensating for the increased length of the paths through the atmosphere. For observations to the east there is no reduction in the path distances when going to lower elevation angles, thus giving increased residual ASD for the lower elevation angles. For symmetry reasons the results presented for observations to the east will also apply for similar observations to the west.

\section{B. Pointing close to the sun}

During a solar conjunction the spacecraft $(\mathrm{S} / \mathrm{C})$ is crossing the sky close to the sun, and in order to avoid solar radiation from corrupting the data set, the MWR needs to be pointed off the S/C direction. A minimum angular distance between the MWR and the sun, $\delta_{M W R}=3.3^{\circ}$ was selected in this study because of the results shown in [8]. This value is three times the modeled MWR beam width. Also the DSA needs a margin against pointing directly to the sun. A limit $\delta_{D S A}=2.5^{\circ}$ has been set to agree with the operational limitations present during the Cassini SCE1 experiment [18]. During this experiment the NASA Deep Space Station (DSS) used was pointed no closer than 5 solar radii from the sun, characterized by an average diameter of $0.5 \mathrm{deg}$.

The limits $\delta_{M W R}$ and $\delta_{D S A}$ form a region in the plane of the sky surrounding the sun to which the DSA can point, but the MWR cannot, see Fig. 5. We simulated possible MWR calibration performance when the DSA was pointed as close to the sun as allowed. Four different cases of single MWR calibration were investigated. In these cases the MWR was pointing as close as possible to the DSA direction. We also studied four cases using two MWRs, either pointing aligned symmetrically around the DSA pointing, or pointing to form equilateral triangles. The eight cases are illustrated in Fig. 5, and the offsets between DSA and MWR pointing directions are given in Table II. 


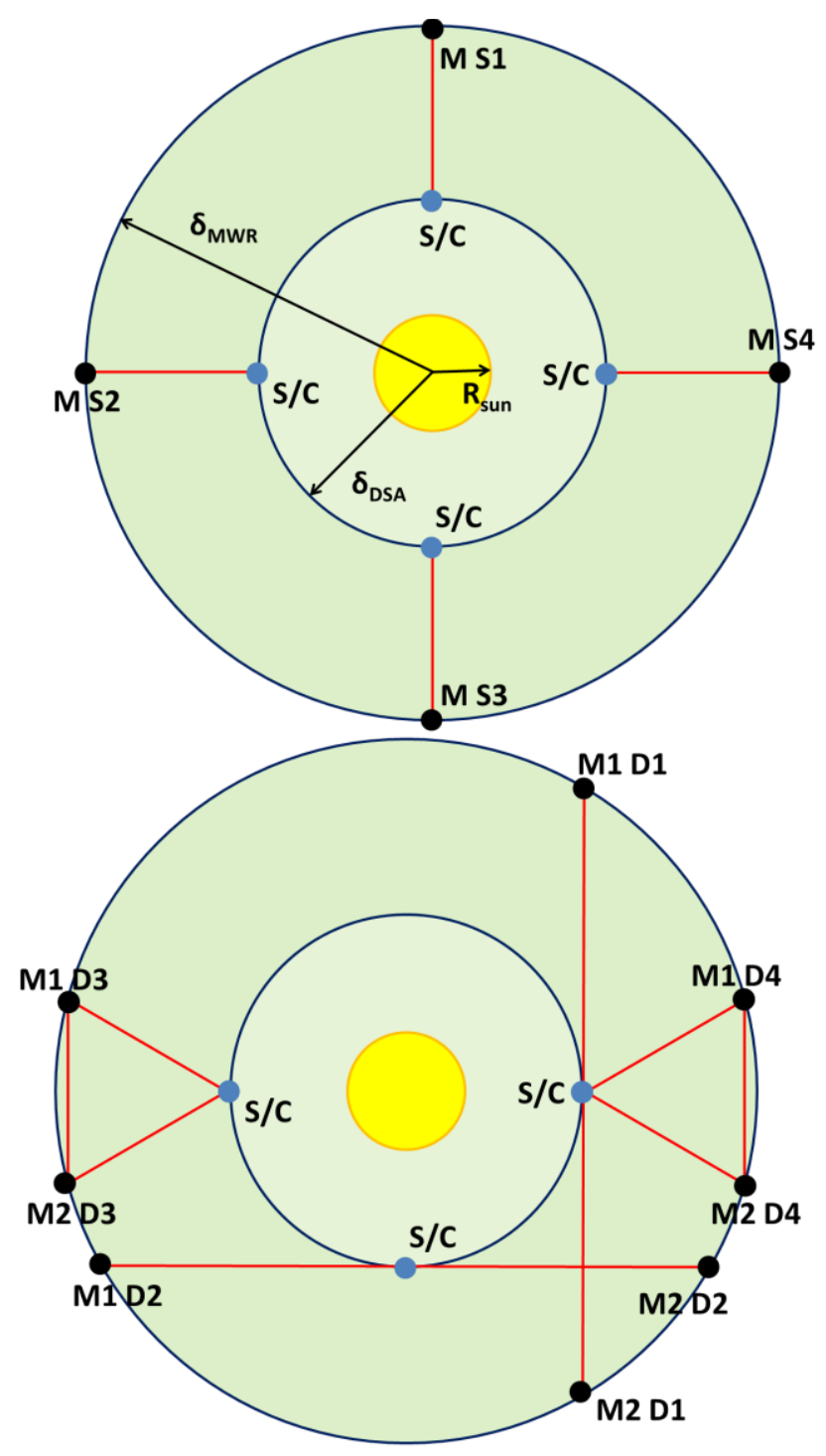

Fig. 5 Illustrations of the region in the plane of the sky surrounding the sun (central circle with radius $\mathrm{R}_{\text {sun }}$ ) to which neither DSA nor MWRs are allowed to point (inner area with radius $\delta_{\mathrm{DSA}}$ ), and the region to which the DSA can point, but an MWR cannot (outer area with radius $\delta_{\mathrm{MWR}}$ ). In the upper chart four single MWR calibration cases, S1-S4, are depicted with the spacecraft position (S/C) and the MWR (M). In the lower chart we show four cases, D1D4, with dual MWRs (M1 and M2).

\section{TABLE II. POINTING OFFSET CONFIGURATIONS}

\begin{tabular}{|c|c|c|c|}
\hline $\begin{array}{c}\text { Study } \\
\text { Case }\end{array}$ & $\begin{array}{c}\text { Pointing } \\
\text { Configuration }\end{array}$ & Pointing Offset $\left({ }^{\circ}\right)$ & Description \\
\hline $\mathrm{S} 1$ & Boundary Up & \multirow{4}{*}{2.05} & \multirow{4}{*}{$\begin{array}{l}\text { One MWR follows the } \\
\text { S/C at the minimum } \\
\text { angular distance }\end{array}$} \\
\hline S2 & Boundary East & & \\
\hline S3 & $\begin{array}{l}\text { Boundary } \\
\text { Down }\end{array}$ & & \\
\hline S4 & $\begin{array}{l}\text { Boundary } \\
\text { West }\end{array}$ & & \\
\hline D1 & $\begin{array}{l}\text { Symmetric } \\
\text { Vertical }\end{array}$ & \multirow{2}{*}{3.22} & \multirow{2}{*}{$\begin{array}{l}\text { Two MWRs are aligned } \\
\text { with the S/C position } \\
\text { symmetrically }\end{array}$} \\
\hline $\mathrm{D} 2$ & $\begin{array}{l}\text { Symmetric } \\
\text { Horizontal }\end{array}$ & & \\
\hline D3 & $\begin{array}{l}\text { Equilateral } \\
\text { East }\end{array}$ & \multirow{2}{*}{2.09} & \multirow{2}{*}{$\begin{array}{l}\text { Two MWRs pointing } \\
\text { positions and the } \mathrm{S} / \mathrm{C} \\
\text { position create an } \\
\text { equilateral triangle }\end{array}$} \\
\hline D4 & $\begin{array}{c}\text { Equilateral } \\
\text { West }\end{array}$ & & \\
\hline
\end{tabular}

The resulting ASD for the eight cases when pointing to the east at different elevation angles are presented in Fig. 6 for short observation time, $20 \mathrm{~s}$, and in Fig. 7 for long, $1000 \mathrm{~s}$, observation time. For comparison we have included results for no pointing offset using a single MWR, S0, and dual MWRs, D0.
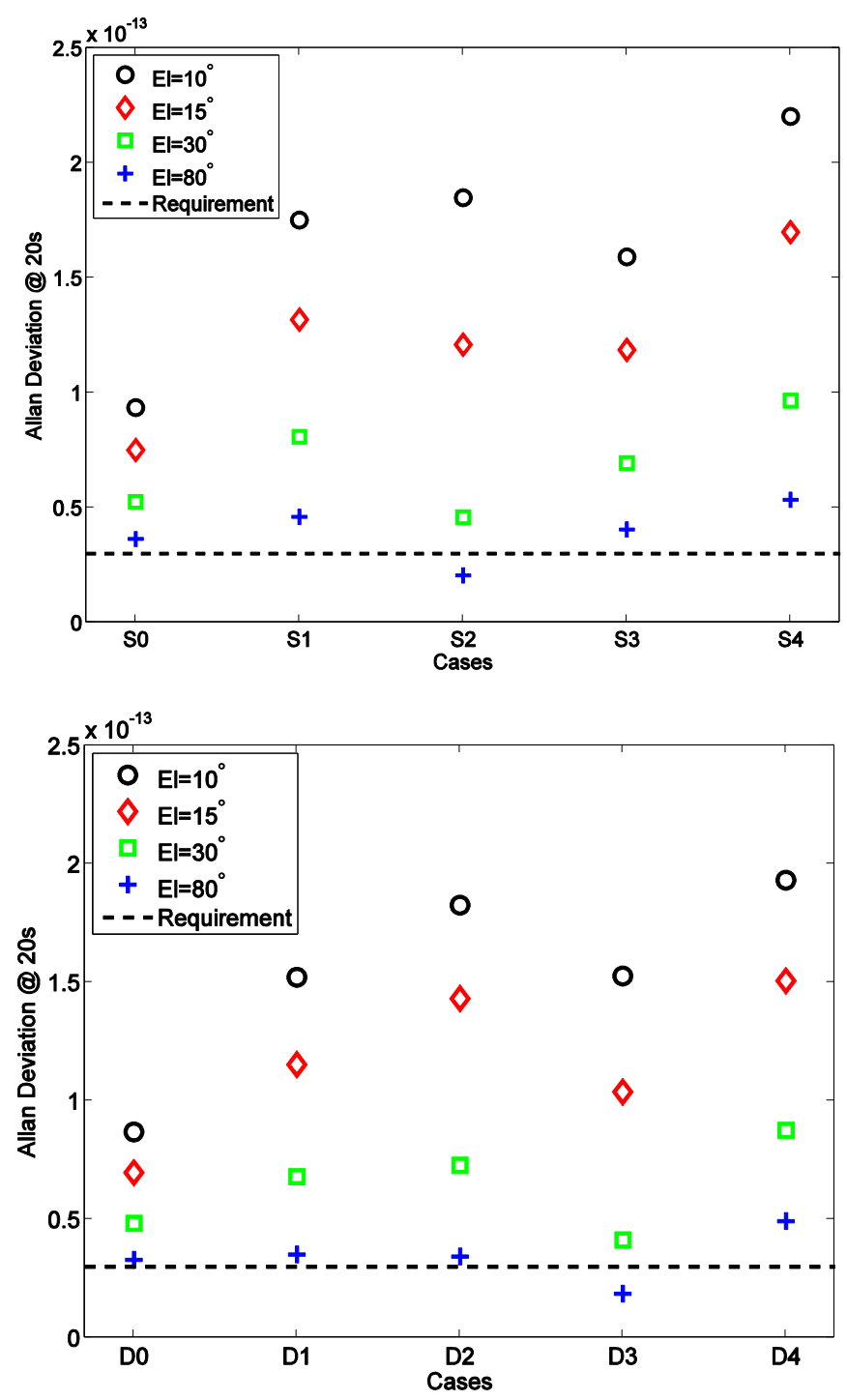

Fig. 6 The residual ASD when calibrating the wet delay of the DSA pointing close to the sun with data from one (top graphs) or two (bottom graphs) MWR(s). The eight cases of calibration, S1-S4 and D1-D4, are defined in Figure 5 and Table II. The corresponding results for pointing in the same direction as the DSA ( $\mathrm{S} 0$ and D0), as well as the design requirement, have been included in the graphs. They are referred to the $20 \mathrm{~s}$ integration time results. 

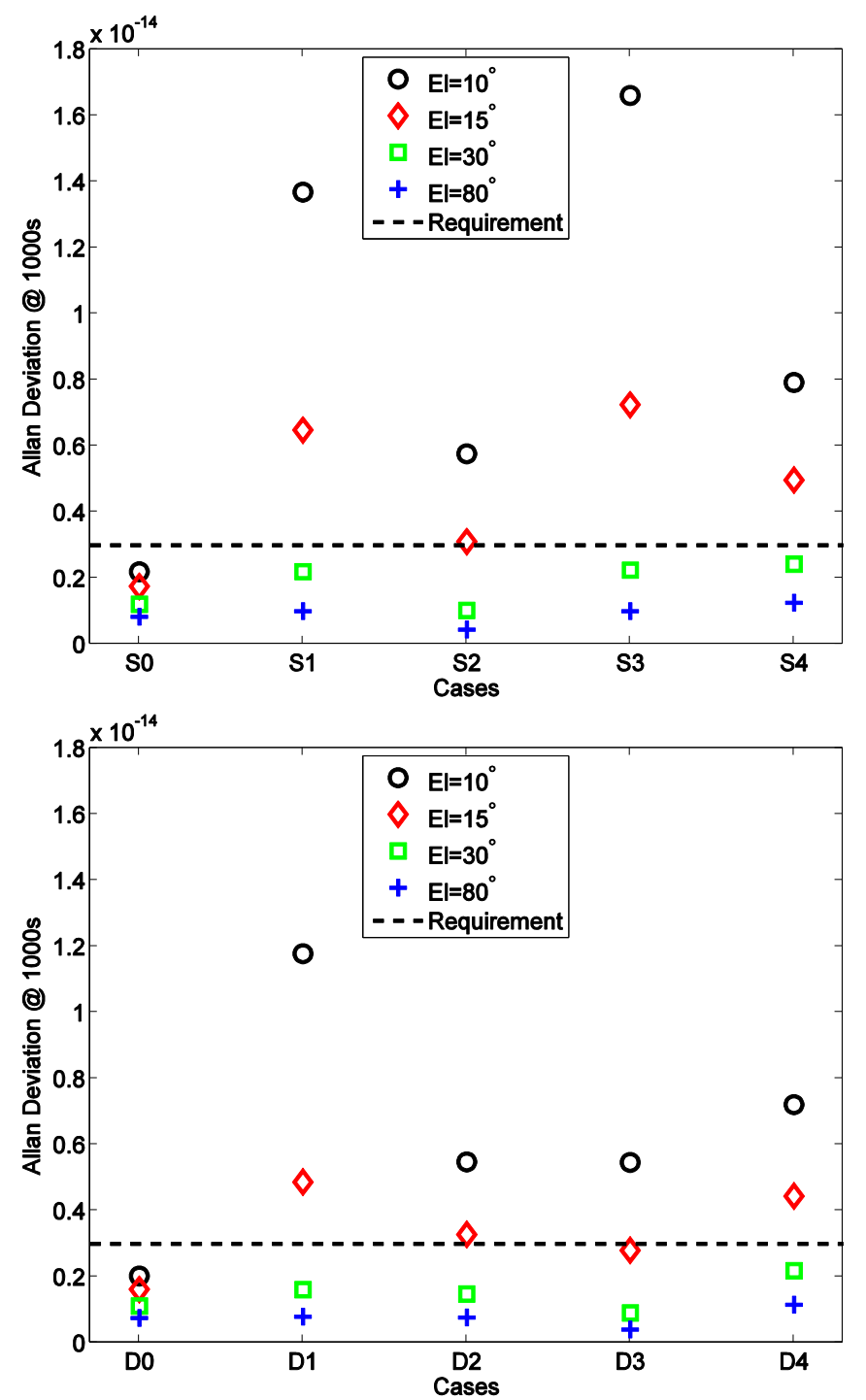

Fig. 7 The residual ASD when calibrating the wet delay of the DSA pointing close to the sun with data from one (top graphs) or two (bottom graphs) MWR(s). The eight cases of calibration, S1-S4 and D1-D4, are defined in Figure 5 and Table II. The corresponding results for pointing in the same direction as the DSA (S0 and D0), as well as the design requirement, have been included in the graphs. They are referred to the $1000 \mathrm{~s}$ integration time results.

It is clear from Fig. 6 and Fig. 7 that the off-pointing in general gives a significant increase in the ASD for the residual wet delay. This is especially pronounced at lower elevation angles. An expected benefit from using two (ideal) MWRs instead of one is found to be limited. When using the two MWRs in a symmetric alignment (cases D1 and D2) a relatively large angular offset is needed, thereby reducing the calibration quality. For the equilateral triangle configurations (D3 and D4) the two MWRs point to fairly similar points on the sky, and do not provide significantly better calibration than what a single MWR would do. Therefore the result for case D3 is fairly similar to what its single MWR counterpart, S2, give, and also D4 and S4 results agree. It should be remembered that the simulations preformed only address the geometrical aspects of wet delay calibrations using MWRs; the combination of data from two MWRs will in general reduce the contribution from instrumental noise, and could also be vital from the redundancy point of view.

For cases S2 and D3 the performance at higher elevation angles ( $30^{\circ}$ and above) is better than those with no pointing offsets ( $\mathrm{S} 0$ and $\mathrm{D} 0$ ). This is a consequence of the MWR beams to some extent cross the DSA beam. The DSA points to the east in this simulation, while the MWR beam starts on the ground from a point south of the DSA and ends up to the north on the sky, due to the pointing offset. In this case the average distances between points in the DSA beam and the MWR beam will be smaller than what is the case when the two beams are parallel. This effect will be studied further below.

\section{Mitigation using crossing beams}

The importance of small distances between the air volumes of atmospheric variability in the DSA and MWR beams, as described above, lead to the idea of introducing a deliberate pointing offset in order to compensate for the distance created by the position offset; i.e. we can obtain a positive impact from letting the DSA and MWR beams cross each other. We made a set of simulations where the DSA pointed to the east and a single MWR (south of the DSA) also point approximately to the east, but with a small azimuth offset introduced. The east direction was selected in these simulations since the MWR calibration in this direction could have significantly larger residual ASD than observations to the south in the standard configuration presented above.

The results for a set of elevation angles are presented in Fig. 8. In each graph the ASD is scaled with the ASD for no pointing offset, i.e. the relative value equals 1 for pointing offset of zero.
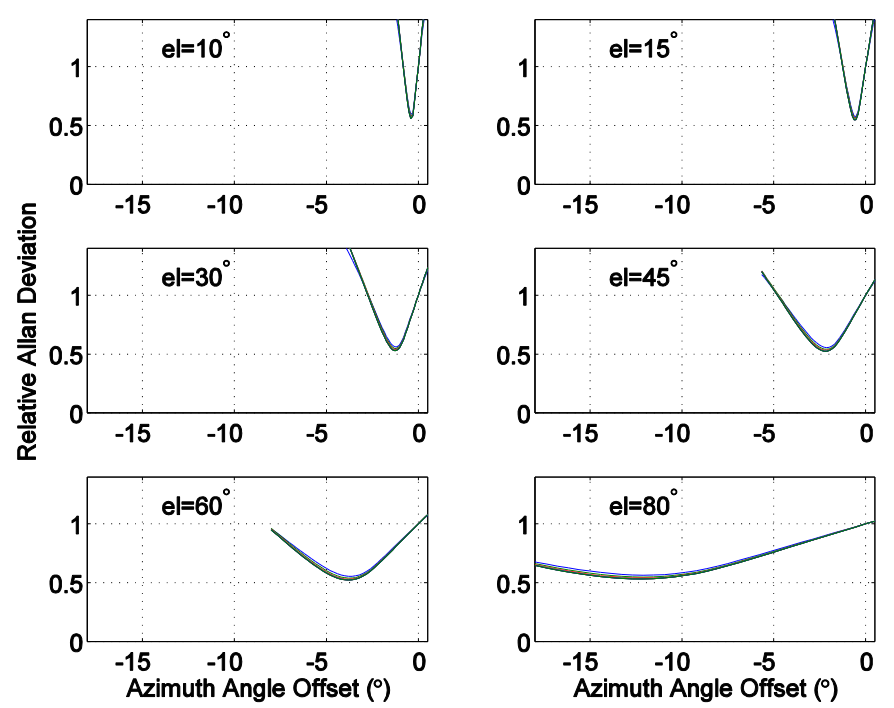

Fig. 8 The residual ASD when the wet delay of the DSA, pointing to the east, is calibrated using an MWR located $25 \mathrm{~m}$ to the south of the DSA. Varying degree of azimuth offset is introduced for the MWR. All results are scale with the zero offset result. In each graph the individual (almost identical) curves for nine different integration times, between $20 \mathrm{~s}$ and $10000 \mathrm{~s}$, are drawn.

For all elevation angles investigated a minimum in the relative ASD of about 0.55 is found, i.e. a pointing offset can reduce the ASD to approximately $55 \%$ of its value for no 
offset. The azimuth offset yielding the minimum showed to correspond to the DSA beams crossing at a height of $650-700$ $\mathrm{m}$ for all elevation angles simulated, i.e. at $65-70 \%$ of the 1 $\mathrm{km}$ planetary boundary layer assumed in the simulations. At lower elevation angles the optimal beam crossing point (at a height of 650 to $700 \mathrm{~m}$ ) will be located further away from the antennas, thereby leading to a smaller great-circle-angle between the antenna pointing directions. At higher elevation angles the crossing point is closer, thus requiring larger greatcircle-angle offsets. At the same time, at higher elevation angles a greater azimuth angle offset is required to achieve a certain great-circle-angle offset. This leads to the increase in azimuth angle offset for optimal performance seen in Fig. 8 . The resulting ASD for the optimal offsets has been compiled in Fig. 9.

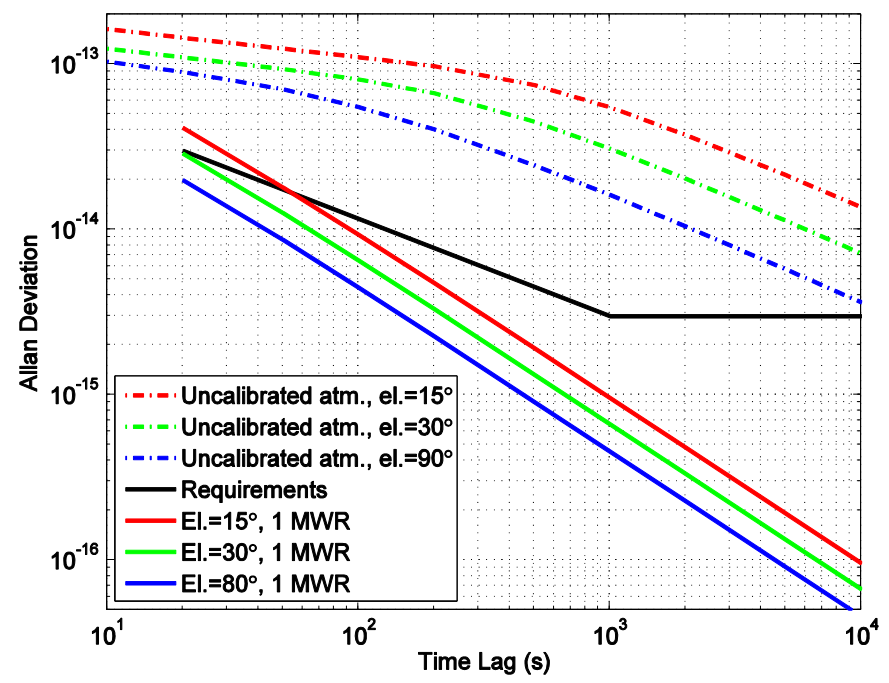

Fig. 9: The residual ASD for the wet delay of the DSA pointing to the east when calibrated using one MWR with deliberate pointing offset. The MWR is located $25 \mathrm{~m}$ south of the DSA and the offset is such that the DSA and MWR beams are crossing at approximately $67 \%$ of the path through the atmosphere. The legend reflects the curve order top-down at $1000 \mathrm{~s}$ integration time.

This graph can be compared with the graph Fig. 3. The significant reduction in residual ASD with the deliberate pointing offset has led to a significant increase in the probability to meet the design requirement also for short integration times.

With one MWR at the site there are situations when an offset optimized in the way presented here would lead to MWR observations too close to the sun. With two MWRs and at a large enough separation the chance of being able to point at least one of them with a beneficiary offset increases. A thorough optimization of locations and pointing directions for two MWRs with the constraint of avoiding interference from both the sun and the DSA structure is a challenge for future further studies.

\section{CONCLUSIONS}

The simulation results demonstrate that the variations in the atmosphere cause a significant contribution to the MCS error budget, even when deploying one or two MWR(s) as close as possible to the DSA. The design requirements on ASD of the residual wet delay are especially challenging for shorter integration times, say, 20-200 s. We present a procedure to mitigate the residual ASD due to the different positions on the ground of the MWR(s) and DSA. By introducing a pointing offset for the MWR beam such that it crosses the DSA beam the distance between points in the air volumes decreases, and hence does the residual ASD. For the case studied the resulting ASD was reduced to $55 \%$ of its values for zero pointing offset. In the simulations the turbulence strength parameter, $C_{n}^{2}$, was set to be constant with height up to $1 \mathrm{~km}$, and above that set to zero. The point of crossing for the optimal ASD occurred at a height of $650-700 \mathrm{~m}$, corresponding to $65-70 \%$ of the maximum height, $1 \mathrm{~km}$. The optimal height for the beams crossing point will, of course, depend on the actual height distribution of variations in the water vapor content. A thorough analysis of this distribution, accompanied by refined simulations, could hence guide the design of MWR pointing schemes.

There are natural variations with time in the parameters describing these variations, e.g., $C_{n}^{2}$. This means that there are occasions where the requirements are relatively easily met, also for shorter integration times, as well as occasions with much smaller chance of meeting the requirements. It is therefore essential to have means to estimate the size of atmospheric variability during operation in order to assess the quality of the present wet delay calibration data. This calls for either algorithms to derive variability parameters from the MWR data themselves, or the inclusion of other dedicated sensors in the MCS.

\section{REFERENCES}

[1] Bar-Sever Y. E., C. S. Jacobs, S. Keihm, G. E. Lanyi, C. J. Naudet, H.W. Rosenberger, T. F. Runge, A. B. Tanner, and Y. Vigue-Rodi, "Atmospheric media calibration for the deep space network," Proc. IEEE, 95 (11), pp. 2180-2192, 2007.

[2] Benkhoff J, J. van Casteren, H. Hayakawa, M. Fujimoto, H. Laakso, M. Novara, P. Ferri, H. R. Middleton, R. Ziethe, "BepiColomboComprehensive exploration of Mercury: Mission overview and science goals", Planetary Space Sci., 58 (1-2), pp. 2-20, ISSN 0032-0633, 10.1016/j.pss.2009.09.020, 2010.

[3] Iess, L., S. Asmar, Tortora, P. "MORE: An advanced tracking experiment for the exploration of Mercury with the mission BepiColombo", Acta Astronautica, 65 (5-6), pp. 666-675, DOI:10.1016/j.actaastro.2009.01.049, 2009.

[4] Alappattu D. P., and P. K. Kunhikrishnan, "First observations of turbulence parameters in the troposphere over the Bay of Bengal and the Arabian Sea using radiosonde", J. Geophys. Res., 115, D06105, doi:10.1029/2009JD012916, 2010.

[5] Nastrom, G. D., and F. D. Eaton, "Relationship between refractivity turbulence intensity from MST radar observations and synoptic-scale vorticity", Radio Sci., 44, RS6003, doi:10.1029/2008RS004104, 2009.

[6] Jacobson, A.R. and R. Sramek, "A method for improved microwaveinterferometer remote sensing of convective boundary layer turbulence using water vapor as a passive tracer", Radio Sci., 32 (5), 1851-1860, 1997.

[7] Nilsson, T., and R. Haas, "Impact of atmospheric turbulence on geodetic very long baseline interferometry", J. Geophys. Res., 115, B03407, doi:10.1029/2009JB006579, 2010.

[8] Nilsson, T., J. L. Davis, and E. M. Hill, "Using ground-based GPS to characterize atmospheric turbulence", Geophys. Res. Lett., 36, L16807, doi:10.1029/2009GL040090, 2009. 
[9] Nastrom, G. D., and F. D. Eaton, "Seasonal variability of turbulence parameters at 2 to $21 \mathrm{~km}$ from MST radar measurements at Vandenberg Air Force Base", California, J. Geophys. Res., 110, D19110, doi:10.1029/2005JD005782, 2005

[10] Kao, C.-Y. J., D. I. Cooper, J. M. Reisner, W. E. Eichinger, and M. Ghil, "Probing near-surface atmospheric turbulence with high-resolution lidar measurements and models", J. Geophys. Res., 107(D10), 4081, doi:10.1029/2001JD000746, 2002

[11] Avila, R., and J. Vernin, "Turbulence profiles with generalized scidar at San Pedro Martir observatory and isoplanatism studies", Publ. Astron. Soc. Pacific, 110, 1106-1116, 1998

[12] Tanner A. B., Riley A. L. "Design and performance of a high-stability water vapor radiometer", Radio Sci., 38 (3), 8050, doi:10.1029/2002RS002673, 2003.

[13] Davis J.L., T.A. Herring, I.I. Shapiro, A.E.E. Rogers, G. Elgered, Geodesyby radio interferometry: effects of atmospheric modeling errors on estimates of baseline length. Radio Sci., 20:1593-1607, doi:10.1029/RS020i006p01593, 1985.

[14] Schween J.; S. Crewell; U. Loehnert; A. Graziani; P. Tortora; A. Martellucci; M. Mercolino; T. Rose; "Microwave Radiometers for Deep Space radioscience experiments: Instrumental internal noise characterization," Microwave Radiometry and Remote Sensing of the Environment (MicroRad), 2012 12th Specialist Meeting on, pp.1-4, 5-9 March 2012, doi: 10.1109/MicroRad.2012.6185269

[15] Allan D.W., "Statistics of Atomic Frequency Standard", Proc. IEEE, 54, (2), 221-231, 1966.

[16] Tortora P., S. Crewell, G. Elgered, A. Graziani, P. Jarlemark, U. Loehnert, A. Martellucci, M. Mercolino, T. Rose and J. Schween, "AWARDS: Advanced Microwave Radiometers In Deep Space Stations", Space Commun. Vol. 22, Number 2-4, (2009-2013) 159-170, November 2013

[17] Treuhaft, R. N., and G. E. Lanyi, "The effect of the dynamic wet troposphere on radio interferometric measurements", Radio Sci., 22 (2), 251-265, doi:10.1029/RS022i002p00251, 1987.

[18] Tortora P., A. Graziani, G. Elgered, P. Jarlemark, S. Crewell, U. Loehnert, and T. Rose, "AWARDS: Advanced Microwave Radiometers In Deep Space Stations, Technical Report 12, Review of algorithms, models, technology, and experimental techniques relevant for the MORE MCS” ESTEC Contract No. 23127/10/NL/LvH, available from http://publications.lib.chalmers.se/, 2011

[19] Bertotti B., L. Iess and P. Tortora, "A test of general relativity using radio links with the Cassini spacecraft", Nature, 425, pp. 374-376, 2003.

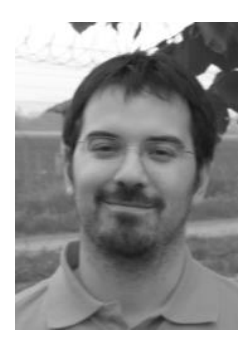

Alberto Graziani was born in Cotignola, Italy, in 1980. He received the B.S. and M.S. degrees in aerospace engineering from the University of Bologna, Forlì, Italy, in 2003 and 2006, respectively, the Ph.D. degree in industrial engineering from University of Bologna, Forlì, Italy, in 2010. In 2009, he was a stagier at the European Space Agency. He is currently a postdoc researcher at the University of Bologna, Forlì, Italy. His research focus on the deep space missions for planetary exploration, in particular his work is in the calibration of transmission media with GNSS and radiometry systems. In 2008-2012 he was member of the COST IC0802.

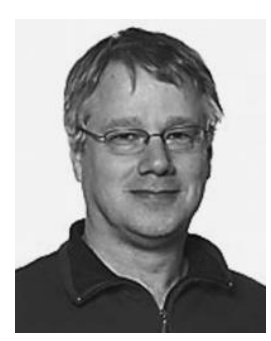

Per Jarlemark Per Jarlemark was born in Hjo, Sweden, in 1962. He received the M.Sc. degree in engineering physics from Uppsala University, Uppsala, Sweden, in 1986 and the Ph.D. degree in electrical engineering from the Chalmers University of Technology, Göteborg, Sweden, in 1997. Since 1999, he has been with the SP Technical Research Institute of Sweden, Borås, Sweden. His research interests are in the area of measurement technology and include space geodetic techniques and microwave radiometry for propagation delay derivation

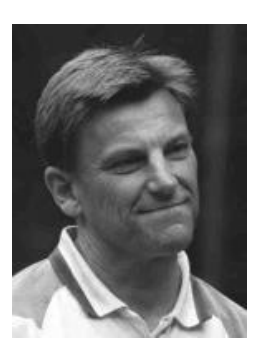

Gunnar Elgered was born in Götene, Sweden, in 1955. He received the M.S.E.E and Ph.D. degrees from Chalmers University of Technology, Gothenburg, Sweden, in 1977 and 1983, respectively. $\mathrm{He}$ is presently a Professor in electrical measurements and chairs the Department of Earth and Space Sciences at Chalmers. His research is focused on remote sensing of the atmosphere using space geodetic techniques and microwave radiometry.

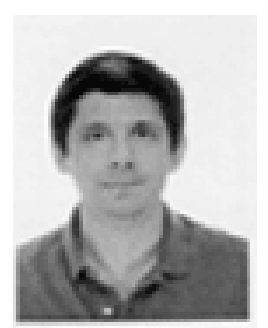

Antonio Martellucci received the Laurea degree in electrical engineering and the $\mathrm{Ph} . \mathrm{D}$. degree in applied electromagnetics from the University of Rome "La Sapienza," Rome, Italy, in 1987 and 1992, respectively.

In 1988, he joined Selenia Group (now part of Finmeccanica) as an Optical Engineer where he worked on the development of optical active systems. From 1989 to 2000, he worked at the Fondazione Ugo Bordoni "Radio communication Systems Division," Rome, as a researcher on atmospheric propagation effects for terrestrial and spatial radio communication systems. He also took part in European COST 210 and 255 projects and various ESA projects on rain scatter, clear air propagation modelling and climatological databases. In 2001, he joined the European Space Agency, ESA-ESTEC, The Netherlands, Directorate of Technical and Quality Management, as a Radiowave Propagation Engineer where he is currently involved in ESA Telecommunication (ARTES and Alphasat), Navigation (Galileo), Earth Observation (ENVISAT) and Science (Gaia, Bepi Colombo) Programmes. At ESA, he is currently involved in models for multimedia SatCom systems, including fade mitigation techniques, modelling and characterization of tropospheric effects for navigation systems and development of ground propagation equipment. He is author of more than 90 publications in books, international journals and conference proceedings.

Dr. Martellucci was a recipient of the Young Scientist Award of XXV URSI General Assembly in 1996. He has been the general Editor of the EU COST 255 and since 2001 he was member of the COST 280 Management committee. In 20082012 he was chairman of the COST IC0802. He is also member of the ESA delegation at ITU-R SG3."

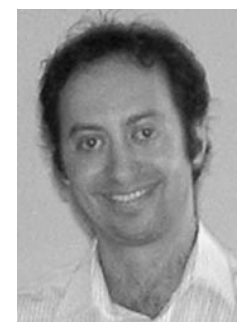

Mattia Mercolino received the M.S. degree in aerospace engineering from the University of Rome "la Sapienza", Rome, Italy, in 2002. From 2002 to 2004, he has been collaborating with the Aerospace and Astronautic Engineering Department, University of Rome "la Sapienza", where he worked on the radio science experiments onboard Cassini and SMART-1 missions 
and on the proposal for the selected MORE radio science experiment onboard the Bepi Colombo ESA cornerstone mission to Mercury. In 2004, he joined the European Space Operations Centre, European Space Agency to work on deltaDOR and radio science. He is currently part of the Ground Antennas Systems section, providing support to interplanetary missions and currently in charge of the delta-DOR system.

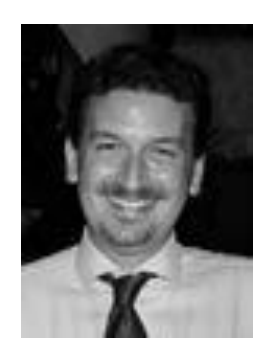

Paolo Tortora (M'99) received the Laurea Degree in Aeronautical Engineering and the Ph.D. in Aerospace Engineering from the University of Rome "la Sapienza", Italy, in 1997 and 2001 respectively. Since 2002 he has been associated with the University of Bologna, where he leads research activities ranging from small satellite missions and applications to radio science experiments on deep space probes and interplanetary navigation. He is currently an Associate Professor in Astronautics \& Space Systems, leads the "Microsatellites and Micro Space Systems" and "Radio Science and Planetary Exploration" laboratories and, since 2012, chairs the BSc and MSc Degrees Programs in Aerospace Engineering.

Prof. Tortora is a Member of the IEEE, the AIAA and the ION. 